\title{
Influence of crosswise non-homogeneity of particulate flow on residence time distribution in a continuous mixer
}

\author{
V. Mizonov ${ }^{\mathrm{a}, *}$, H. Berthiaux ${ }^{\mathrm{b}}$, C. Gatumel $^{\mathrm{b}}$, E. Barantseva ${ }^{\mathrm{a}}$, Y. Khokhlova ${ }^{\mathrm{a}}$ \\ a Ivanovo Power Eng. State University, Department of Applied Mathematics, Rabfakovskaya 34, 153003 IVANOVO, Russia \\ ${ }^{\mathrm{b}}$ Ecole des Mines d'Albi-Carmaux, RAPSODEE Centre, UMR CNRS2392, Albi, France
}

Keywords:

Mixing

Markov chain

Flow pattern

Tracer

Residence time distribution

\begin{abstract}
A B S T R A C T
The objective of the study is to develop a mathematical model of continuous mixing of granular materials, which takes into account the crosswise non-homogeneity of particulate flow inside a mixer. The target function of modeling is the residence time distribution of a tracer injected into the inflow. The model is built on the basis of the theory of Markov chains. The working space of a mixer is presented as a 2D array of perfectly mixed cells, through which the tracer travels according to the matrix of transition probabilities. The model covers the tendency of the tracer to upward/downward segregation. Finally, for a given flow pattern, the model allows calculating the RTD curves for various throughputs and for various properties of an imperfect tracer.
\end{abstract}

\section{Introduction}

Powder mixing is a very important operation for a wide range of industries, as it fixes the homogeneity of a mixture to a scale at which end-used properties have to be reached. Academic research and industrial development in this area put a lot of important efforts in measuring these properties and attempts to model the motion of the particles.

In this paper, we will focus on developing a mathematical model based on application of the theory of Markov chains to describe solids mixing in continuous mixers of different kind. The essence of mixing itself is migration of particles, individually or as blocks of particles, from one zone of operating volume to another. The essence of the theory of Markov chains is to describe "migration" of probabilities from one state to another. The matrix of transition probabilities is a sort of mixer acting in the sample space and transforming it. It looks like the very process and the very tool were created for each other. However, a lot of theoretical and experimental work is to be done in order to set up correspondence between a mixer and its matrix image. The basic operators of the approach and some of ideas on restoration of the matrix of transition probabilities can be found below.

This approach to model the process has already been used by various authors. For example, in the work by Wang, R.H. and Fan, L.T. [1] it was applied to model the state of mixture after passing a static mixer. However, the approach misses the evolution of process parameters, and physical features of mixing zone are not presented. In further works by these authors [2] the model of the process, in which

\footnotetext{
* Corresponding author.

E-mail addresses: mizonov@home.ivanovo.ru (V. Mizonov), berthiau@enstimac.fr (H. Berthiaux).
}

transitions only to the neighboring cells were allowed, was developed. Here, not only does the model answer the question what the final state is to be but also how it has been formed. However, seems that now it can be said that the 1D chain models have already exhausted their potentialities, and some new ideas should be introduced to improve the predictability of the models.

The general strategy of application of the theory of Markov chains to model various processes in chemical engineering and powder technology is described in [3] and also in our previous papers [4-6]. Here we are planning to concentrate on some particular problems of application of the theory to examine the influence of crosswise nonhomogeneity of particulate flow inside a continuous mixer at different hold-ups and specific properties of a tracer on the most informative characteristic of the process - the residence time distribution of tracer particle (RTD). It is known that the RTD curves for real mixers can differ strongly from ones obtained from solutions to 1D dispersion equation, or from 1D chain models. The talk about crosswise nonhomogeneity automatically presupposes that the model is to be developed to a multi-dimensional case, at least to a 2 dimensional one. Namely this is the objective of the study described below.

\section{Two-dimensional cell model of mixing: model structure and governing equations}

As it was mentioned above, the general rules of constructing 2D chains models can be found in [4-6]. Here we shall concentrate on the $2 \mathrm{D}$ model, which is to answer the questions formulated above. The cell presentation of the operating volume of the continuous mixer and the particulate flow pattern is shown in Fig. 1.

The volume is presented as the mxn array of cells where $n$ is the number of columns of cells in the longwise direction, and $\mathrm{m}$ is the 


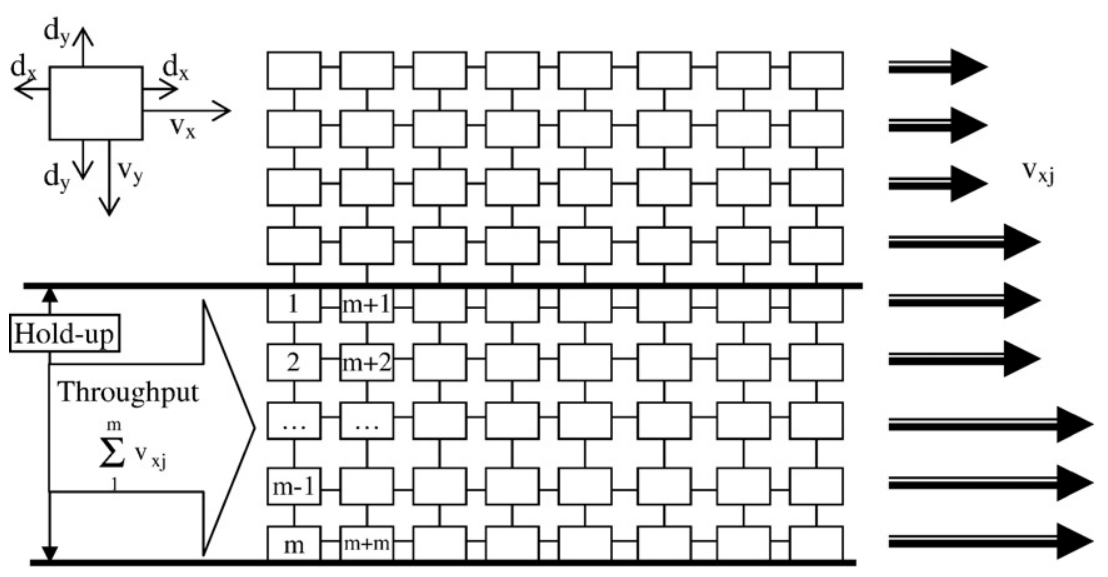

Fig. 1. The cell model of operating volume and the flow pattern at the given hold-up.

number of rows of cells in the crosswise one. The number of columns (the longwise direction) is fixed; the number of rows is also fixed for a certain flow regime but can vary from one flow regime to another. In fact, the number of rows $m$ represents the hold-up, which can vary only discretely in the model involving row by row of the array. It is also supposed that each layer of material (each row of cells) has its predominant mean velocity, which forms the axial transition probability and is unknown in advance. However, it is considered that the velocity does not vary with the presence, or absence of layers above. The dimensionless value of corresponding throughput is:

$Q(m)=\sum_{1}^{m} v_{x j}$

where $m$ is the analogue of the hold-up. Eq. (1) allows restoration of the crosswise non-homogeneity of a real flow using the experimental correlation "hold-up versus throughput". Some illustration of this will be shown below.

Let us observe the state of the process in discrete moments of time $t_{i}=(i-1) \Delta t$ where $\Delta t$ is the transition duration, $i=1,2, \ldots$ is its number.

The sample space for the chain shown in Fig. 1 is the set of probabilities of a tracer to occupy cells of the chain. It can be presented as the column state vector of the size $(m \times n) \times 1$, in which the probabilities are placed according to the numeration of the cells shown in Fig. 1:

$S^{i}=\left[S_{1}^{i} S_{2}^{i} \ldots S_{m-1}^{i} S_{m}^{i} S_{m+1}^{i} \ldots S_{m+m}^{i} \ldots S_{n m-1}^{i} S_{n m}^{i}\right]^{t}$

where $t$ means transposing a vector.

During the $i$-th transition the state vector changes and becomes $S^{i+1}$. The sequence of the state vectors is formed by the matrix equality:

$S^{i+1}=P S^{i}$

where $P$ is the matrix of transition probabilities, which is the matrix of the size $(n \times m) \times(n \times m)$. The structure of the matrix is based on the following rule. Each column of it belongs to one of the cells according to this cell number. The probability to transit during $\Delta t$ to a cell is to be placed in the row with the number of this cell. The probability to stay within the cell during $\Delta t$ is placed on the main diagonal of the matrix and is equal to one minus the sum of all the rest non-zero probabilities in this column. For example, for the cell $(m+2)$, which occupies the $(m+2)$-th column of the matrix:

$P_{(m+2),(m+2)}=1-\left(P_{2,(m+2)}+P_{(2 m+2),(m+2)}+P_{(m+1),(m+2)}+P_{(m+3),(m+2)}\right)$.

The last column of the chain belongs to the absorbing state. For this column all the probabilities to stay are equal to 1 , all the rest being equal to zero.
In order to give the transition probabilities more physical sense let us separate from them the completely random transitions and transport transitions like it is shown in the upper left corner of Fig. 1 with these transitions nomenclature. It is obvious that a flow pattern is connected with the distribution $v_{x j}$. As far as a granular material cannot segregate within itself, $v_{y j}$ is related only to a tracer. For the perfect tracer $v_{y j}=0$, and all the other probabilities coincide with ones for the material. They can be different for the imperfect tracer. In particular, if $v_{y j}>0$, the tracer has the tendency to downward segregation, and, if $v_{y j}<0$, it has the tendency to the upward one.

In order to start the computational procedure given by Eq. (3) the initial state vector is to be known. Let us suppose that we introduce the unit portion of a tracer across the inflow. In this case the initial state vector can be written as:

$S^{0}=\frac{1}{\sum_{1}^{m} v_{x j}}\left[\begin{array}{llllllll}v_{x 1} & v_{x 2} & \ldots & v_{x m} & 0 & \ldots & 0\end{array}\right]^{t}$

The outflow of the chosen tracer, which gives the residence time distribution, can be calculated as:

$$
E(i)=\sum_{m(n-1)+1}^{m n}\left(S_{j}^{i+1}-S_{j}^{i}\right)
$$

Thus, the proposed model allows calculating the RTD for an arbitrary flow pattern and comparison of the distributions at different hold-ups, which, in turn, are known functions of throughputs for a given flow pattern and vise versa.

\section{Some examples of simulation}

Let us suppose that the flow-field of a granular material looks like it is shown in Fig. 2. The faster motion of lower layers of it has place in blade mixers where the layers covered by rotating blades move faster than the core of the flow. This flow field corresponds to the correlation between hold-up and throughput shown below. The break of the line means that the hold-up reaches the level involving layers of the material with smaller velocity. At each line segment of this piecewise linear approximation the mean flow velocity is inversely as the slope of the graph at this line segment.

The residence time distributions shown in Fig. 2 are related to the perfect tracer. Until the hold-up reaches the value $M=m=4$ the residence time distribution does not depend on the hold-up, and on the throughput respectively. After that the curve of RTD is being deformed and gradually becomes a bi-modal one. Its variance is becoming greater and greater respectively. The mean residence time remains constant and is equal to the mean flow time at all the range of hold-up variation. 


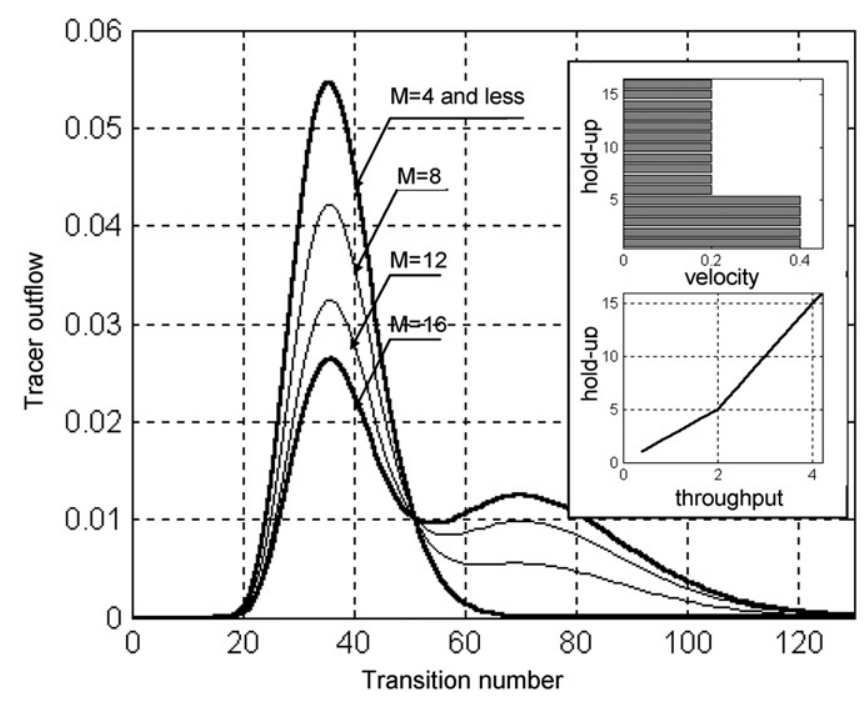

Fig. 2. Influence of flow parameters on the RTD curve for the given flow pattern $\left(d_{x}=d_{y}=0 ; v_{\mathrm{tr}}=0\right)$.

The numerical estimation of how the residence time distribution varies with growing stochastic component of the axial motion of the flow is shown in Fig. 3. The growth of $d_{x}$ at the given hold-up makes the curves of residence time distribution smoother but its variance remains of high value. The similar calculations with respect to the cross-wise dispersion $d_{y}$ show that its influence is much less, and can actually be neglected for realistic flow patterns.

Fig. 4 demonstrates what may happen with the residence time distribution if the tracer is imperfect, i.e., has the tendency to segregation. This tendency is presented by the velocity $v_{y}$, which can be directed upward and backward.

The downward segregation does not affect the residence time distribution in a qualitative sense, and the quantitative changes are not too high. On the contrary, the upward segregation to the area of smaller velocities affects the curves significantly. First the bi-modality moves in, and then the curve transits to the area of higher mean residence times. The important feature of using an imperfect tracer is that the mean residence time at the RTD curve and the mean flow time are not equal. The influence of segregation velocity on the difference is

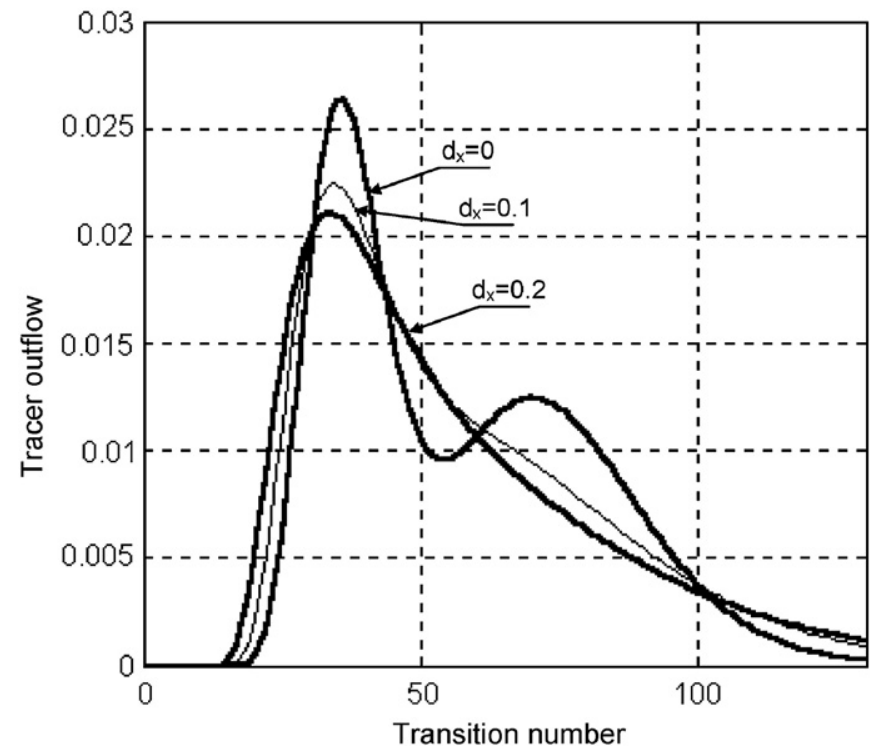

Fig. 3. Influence of axial dispersion of the flow on the RTD curves $\left(M=16 ; d_{y}=0 ; v_{\mathrm{tr}}=0\right)$.

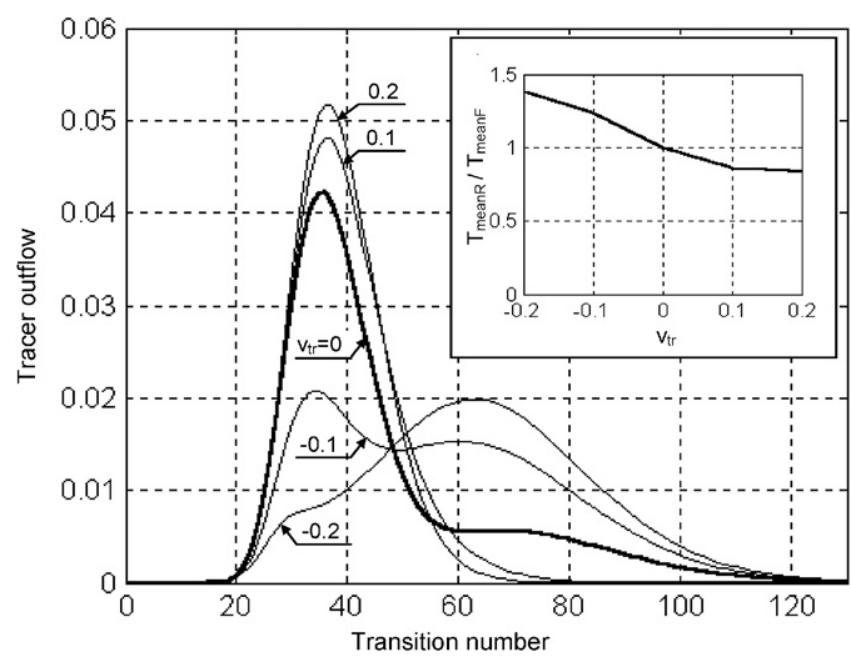

Fig. 4. Influence of tracer segregation on the RTD curves and on deviation of the mean residence time from the mean flow time $\left(M=8 ; d_{x}=d_{y}=0\right)$.

shown in the upper right corner of the figure. It is necessary to emphasize that these conclusions are valid for the given flow field alone: another flow field can give different results but all of them can be estimated using the proposed model.

\section{Experimental validation of the model}

Below some results of experimental validation of the model are shown. The experimental data were obtained at the experimental set with the continuous blade mixer Gericke GCM500. The detailed description of the set, experimental technique and treatment of experimental data can be found in our paper [7]. The comparison of the experimental and calculated data is shown in Fig. 5 where in the upper right corner the experimental correlation between throughput and hold-up is shown. This correlation has clearly expressed piecewiselinear form with the break at the throughput $60 \mathrm{~kg} / \mathrm{h}$, which corresponds to the hold-up equal to $0.6 \mathrm{~kg}$. Namely, this mass of couscous, which was used as the test material, approximately corresponds to the height of the layer covered by the blades of the mixer. After the $60 \mathrm{~kg} / \mathrm{h}$ point the slope of the line is two times as big as before it. It means that the velocity of the core of the material flow is two times as little as in the area covered by blades. The number of layers corresponding to the hold-up at the throughput $60 \mathrm{~kg} / \mathrm{h}$ was taken equal to 5 . Thus, the

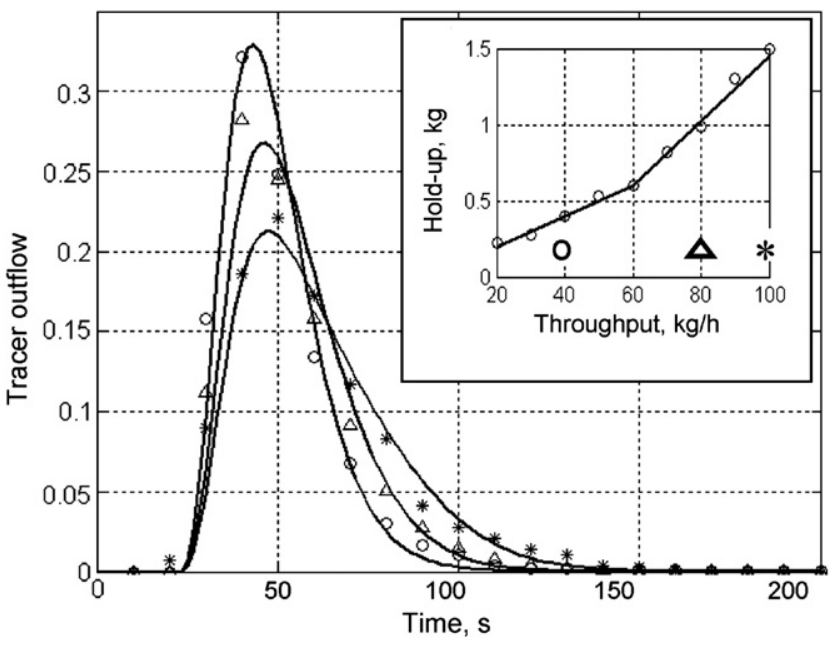

Fig. 5. Comparison of experimental and calculated RTD curves for different throughputs in the mixer Gericke GCM500 at the experimental correlation hold-up versus throughput. Material - couscous, speed of rotor - $60 \mathrm{rpm}$. 
numbers of layers corresponding to 40,80 and $100 \mathrm{~kg} / \mathrm{h}$ were 3,8 , and 13 respectively. Within the range of throughputs less than $60 \mathrm{~kg} / \mathrm{h}$ the curves of residence time distribution practically coincide and only the experimental data for $40 \mathrm{~kg} / \mathrm{h}$ are shown in the graph. The calculated curve for this throughput was adjusted to these data by varying $\mathrm{v}_{\mathrm{x} 1}$ and $d_{x 1}$. The curves for $80 \mathrm{~kg} / \mathrm{h}$ and $100 \mathrm{~kg} / \mathrm{h}$ were calculated without any adjustment using the flow field shown in Fig. 2. It is obvious that there hardly is a model that allows predicting the RTD curve without any calibration of its parameters. However, the proposed model, being once calibrated for one of sets of experimental data on the residence time distribution, allows predicting other various RTD curves for different flow regimes already without any extra calibration.

\section{Conclusions}

The proposed model based on the theory of Markov chains allows estimating how the cross-wise non-homogeneity of the flow of granular material, which is usually the case in blade continuous mixers, influences the residence time distribution. It is shown that the typical for the mixers flow field leads to considerable influence of the throughput (through the hold-up) on the distributions strongly increasing their variance and leading to bi-modality of them at high throughputs. It is also shown that the tendency of an imperfect tracer to downward segregation has little influence on the RTD curves in opposite to the tendency to upward segregation when this influence becomes very strong. Fragmentary experimental validation of the model for the continuous blade mixer Gericke GCM500 has shown that the model has rather high predictability for calculation of the RTD curves under variation of the flow regime.

\section{References}

[1] R.H. Wang, L.T. Fan, Axial mixing of grains in a motionless Sulzer (Koch) mixer, Ind. Eng. Chem. Process Des. Dev. 15 (3) (1976) 381-388.

[2] R.H. Wang, L.T. Fan, Stochastic modeling of segregation in a motionless mixer, Chem Eng. Sci. 32 (1977) 695-701.

[3] A. Tamir, Applications of Markov Chains in Chemical Engineering, Elsevier ed. Amsterdam, 1998.

[4] H. Berthiaux, V. Mizonov, Applications of Markov chains in particulate process engineering: a review, Can. J. Chem. Eng. 85 (6) (2004) 1143-1168.

[5] H. Berthiaux, V. Mizonov, V. Zhukov, Application of the theory of Markov chains to model different processes in particle technology, Powder Technol. 157 (2005) $128-137$.

[6] V.E. Mizonov, et al., Application of multi-dimensional Markov chains to model kinetics of grinding with internal classification, Int. J. Miner. Process 74 (1001) (2004) 307-315

[7] K. Marikh, et al., Flow analysis and Markov chain modelling to quantify the agitation effect in a continuous mixer, Chem. Eng. Res. Des. 84 (A11) (2006) 1059-1074. 